Jean-François Hardy MD, Sylvain Bélisle MD

\title{
Natural and synthetic antifibrinolytics: inert, poisonous or therapeutic agents?
}

"A drug can be an inert substance, a poison, or a therapeutic agent dependent on how it is used and the dosage in which it is given."

ERIC JORPES, PARAPHRASING PARACELSUS

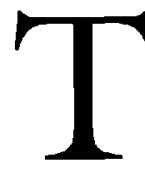

RANSMISSION of infectious diseases and the modulatory effects of allogeneic transfusions on the immune system remain a cause of concern for patients and physicians. More recently, the trend towards a limited access to blood products has jeopardized a number of surgical procedures that may require large amounts of blood. Consequently, numerous strategies are being recommended to decrease or to eliminate the need for transfusion of allogeneic blood products (ABP) specially during and after cardiac surgery. ${ }^{1,2}$ The prophylactic administration of antifibrinolytics, whether they be natural (aprotinin - Trasylol ${ }^{\circledR}$ ) or synthetic (epsilon-aminocaproic acid - Amicar ${ }^{\otimes}$; tranexamic acid - Cyklokapron ${ }^{\circledR}$ ) has received much attention over the past few years. This is probably because their use is simple and intuitively logical. The underlying rationale is to control (ideally, to prevent) the haemostatic defects induced by cardiopulmonary bypass and avoid allogeneic transfusions without having to resort to more complicated and time-consuming methods, such as those involved in autologous blood transfusions. However, despite the apparent homogeneity of the procedures performed, interpretation of the results reported on the use of antifibrinolytics in cardiac surgery remains difficult. Basically, clinicians want to know if they should be administering antifibrinolytics (or which one) to their patients. Before making this decision, readers must determine if the drug is efficacious (based on published data from different randomized clinical trials), if it is likely to be effective in their own clinical context and if its use is efficient for the health care system in which they operate.
When analysing the results of a study on antifibrinolytics, the first question to answer is: does the study focus on the appropriate outcome? The most important outcome to judge the efficacy, effectiveness and efficiency of a drug administered in view of reducing transfusions is the change in the amount of $\mathrm{ABP}$ to which patients are exposed. It must be remembered that the amount of blood shed during and after the operation is only an intermediate outcome. Unfortunately, many studies tend to focus on intermediate outcomes such as bleeding, coagulation profile, platelet count, etc. assuming that any beneficial effect on these will result in a reduction of $A B P$ transfusion. Several studies have reported decreased mediastinal chest drainage with an antifibrinolytic, but no difference in transfusion rates, or did not describe ABP utilization altogether. The clinical relevance of such studies is questionable.

The second question the reader must ask is: were the data analysed appropriately? We want to know the percentage of patients exposed to $A B P$ in the groups under study and, in those patients who received $A B P$, the median number of units and range of $A B P$ administered (since most haemostatic blood products are administered as predetermined quanta, e.g., ten units of platelets, reporting results as mean \pm standard deviation is most often inappropriate). Reporting of data in this manner allows us to determine the risk of being exposed to $\mathrm{ABP}$ and yields information clinicians can easily relate to their own transfusion practice. ${ }^{3}$ Furthermore, non-parametric statistics are, in most cases, desirable since $\mathrm{ABP}$ usage during or after cardiac surgery does not follow a normal (Gaussian) distribution: the majority of patients will receive little or no transfusions, while a few will require huge amounts of red cells and haemostatic blood products.

In this issue of the Canadian Journal of Anaesthesia, Dryden $\varepsilon t$ al. report their experience with tranexamic acid to reduce blood loss and transfusion of $\mathrm{ABP}$ following repeat cardiac valve surgery. Mean duration of 
extracorporeal circulation approached three hours and blood losses were high. In this specific patient population, tranexamic acid effectively reduced the volumes of red cells, plasma and platelets transfused. Unfortunately, as presented, the results do not allow the reader to determine the percentage of patients receiving ABP, nor the number of units to which they were exposed.

We must then examine our own transfusion practice, knowing that it varies widely between institutions. ${ }^{4}$ This may be because surgical blood loss is more important in some centres or because of inappropriate transfusion habits. While antifibrinolytics will not correct the latter, they have been shown repeatedly in several, well controlled studies to decrease surgical blood loss and transfusion of $\mathrm{ABP} .{ }^{5}$ Conversely, when inappropriate transfusion practices are the problem, pertinent protocols for $\mathrm{ABP}$ usage ${ }^{6}$ must be implemented before considering antifibrinolytics. The audit of transfusion practice is an essential prerequisite to optimize the use of $A B P$ in cardiac surgery. ${ }^{7}$

Finally, the choice of a specific antifibrinolytic will be determined by efficacy, incidence of serious complications, and cost. In turn, efficacy depends on patient selection, dose regimen and timing of administration of the drug. Most clinicians agree on the classification of patients at low vs high risk of $\mathrm{ABP}$ usage, even if the classification is imperfect. ${ }^{8}$ Agreement on the most appropriate dose and timing of administration of antifibrinolytics is much more difficult, with the probable exception of aprotinin. Essentially, this is because we know little about the pharmacokinetic and pharmacodynamic behaviour of synthetic antifibrinolytics during CPB. Single or multiple boluses of different sizes, with or without subsequent infusions have been recommended, based on what appears to have been an educated guess at the time the study was elaborated. Innumerable research protocols can be produced by the combination of these variables (patient selection, antifibrinolytic, different dosage regimens, timing of administration), rendering the determination of the most effective therapy almost by chance.

Manipulation of haemostasis is not without risk. Patients present in a hypofibrinolytic state lasting $48 \mathrm{hr}$ after CPB that is characterized by high plasma levels of PAI-1 (plasminogen activator inhibitor type 1$).{ }^{9}$ Such a hypofibrinolytic state is probably beneficial, favouring the conservation of stable haemostatic plugs in injured tissues but, if it were augmented by antifibrinolytics, it could, theoretically, increase the risk of postoperative thrombosis. Somewhat paradoxically, while high-dose aprotinin inhibits fibrinolysis effectively during $\mathrm{CPB},{ }^{10,11}$ it does not exaggerate this late postoperative fibrinolytic shutdown. ${ }^{9}$ On the contrary, lower PAI-1 plasma concentrations in aprotinin treated patients suggest a pro- tective effect of this protease inhibitor on endothelial cells. ${ }^{9}$ The effect of synthetic antifibrinolytics on late postoperative fibrinolysis is unknown, but tranexamic acid administered after CPB and the infusion of protamine was associated with a concerning, albeit not statistically significant, increase in frequency of postoperative myocardial infarction in the study by $\emptyset \mathrm{vrum} e t a l^{12}$

At present, the risk of exaggerating the normal postoperative prothrombotic state with antifibrinolytics is difficult to evaluate and is based on the increased incidence, or absence thereof, of thrombotic complications after cardiac surgery, including myocardial infarction, graft occlusion, cerebrovascular accident, pulmonary embolism and renal failure. Fortunately, these are relatively infrequent events, despite the persistent prothrombin activation after $\mathrm{CPB} .{ }^{13}$ Unfortunately, however, a considerable number of patients is required to detect a difference in the incidence of perioperative thrombotic complications. At present, such numbers may be available only for aprotinin. Extensive pooling of data (totalling 2,283 CABG patients) shows that the full-dose (Hammersmith) regimen is safe and even reduces the incidence of stroke, but suggests that the half-dose regimen may, in fact, increase mortality (from $3.8 \%$ to $5.4 \%$ ). ${ }^{14}$ More than 3,160 patients per group would have been required to detect a $40 \%$ increase in mortality such as that observed for the half-dose regimen. ${ }^{14}$ As for synthetic antifibrinolytics, no study or aggregation of studies published to date has sufficient statistical power to rule out the absence of effect on thrombotic complications after cardiac surgery. ${ }^{5}$

Efficiency of treatment is much more difficult to define and measure. This is a very complex issue that takes into account not only direct costs (cost of the drug and of blood products), but also the ensuing effects of treatment such as length of stay in the operating room, in the intensive care unit and in the hospital; need for surgical re-exploration; treatment of transfusion or drug related complications etc. The expenses involved in administering drugs like EACA and TA arc trivial compared with that for aprotinin. While the initial low cost of synthetic antifibrinolytics is attractive, data on the financial consequences of potential side effects must ensure that these do not outweigh the initial savings. Reduced operating times, less need for work after regular hours, and shorter stays in the intensive care unit and data demonstrating the beneficial effect of antifibrinolytics on transfusion related (or other) complications must become available to justify the cost of the drug in the long term. As mentioned previously, many of the data required to make an enlightened choice are not available at present.

Returning to Paracelsius, what can clinicians conclude from all these studies? First, with the dose regimens in common use today, antifibrinolytics are not 
inert. Second, most studies (as the one presented here by Dryden $e t$ al.) tend to support their therapeutic effect in selected cases where the risk of bleeding after $\mathrm{CPB}$ is high, as part of a more global strategy ${ }^{1}$ to decrease exposure to $\mathrm{ABP}$ associated with cardiac surgery. Third, only large scale studies will be capable of excluding the poisonous effects of synthetic antifibrinolytics. The evolving risks associated with haemorrhage and transfusion of ABP should constantly be balanced against those associated with antibrinolytics, particularly thrombosis. At present, high-dose aprotinin appears to be the least poisonous pharmacological strategy available to reduce the need for ABP after cardiac surgery with extracorporeal circulation.

\section{Les antifibrinolytiques naturels et synthétiques: substances inertes, toxi- ques ou thérapeutiques?}

En paraphrasant Paracelse, Eric Jorpes disait: «Un médicament peut être une substance inerte, toxique ou thérapeutique selon son utilisation et sa dose».

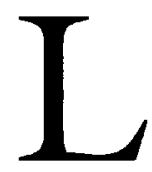

ES patients autant que les médecins redoutent la transmission des infections et les effets modulateurs des transfusions allogènes sur le système immunitaire. Depuis quelque temps, la tendance à limiter l'accès aux produits sanguins compromet un bon nombre d'interventions chirurgicales nécessitant de grandes quantités de sang. En conséquence, on a recommandé des stratégies qui réduisent ou éliminent le besoin de transfuser des produits sanguins allogènes (PSA) surtout pendant et après la chirurgie cardiaque. ${ }^{1,2}$ Ces dernières années, l'administration prophylactique d'antifibrinolytiques a suscité un intérêt considérable, qu'il soient naturels (aprotinine - $\operatorname{Trasylo}^{\circledR}$ ) ou synthétiques (acide epsilon-aminocaproïque - Amicar ${ }^{\otimes}$; acide tranéxamique - Cyclokapron ${ }^{\circledR}$ ). Vraisemblablement, c'est parce que leur mode d'emploi est simple et que le choix est logique. Le but recherché est de contrôler (à défaut de prévenir) la déficience des mécanismes hémostatiques induite par la circulation extracorporelle et d'éviter les transfusions allogènes sans avoir à faire appel à des moyens compliqués et chronophages, comme c'est le cas avec les transfusions de sang autologue. Cependant, malgré l'apparente cohésion des moyens, il demeure difficile d'interpréter les résultats obtenus avec les antifibrinolytiques en chirurgie cardiaque. Au départ, les cliniciens veulent savoir s'ils devraient administrer des antifibrinolytiques (et lesquels) à leurs patients. Avant de prendre une telle décision, le lecteur doit établir si le produit est efficace, (sur la base des données de publications émanant d'essais cliniques aléatoires divers), si cette efficacité s'appliquera à leur propre pratique et si son utilisation sera efficiente dans le système de santé où ils évoluent.

Quand on analyse les résultats d'une étude sur les antifibrinolytiques, une première question se pose : cette étude fait-elle ressortir des résultats pertinents? La quantité de PSA reçue constitue le critère le plus important permettant de juger de l'efficacité, de l'efficience, et de la puissance d'une substance pharmacologique administrée dans le but de diminuer les transfusions. Il faut mentionner que la quantité de sang perdue pendant et après l'intervention n'est qu'un résultat d'étape. Malheureusement, plusieurs études ont tendance à mettre l'accent sur des résultats intermédiaires comme le saignement, le bilan hémostatique, le décompte plaquettaire, etc. en assumant que cette action bénéfique aura pour effet de diminuer le nombre de transfisions. Plusieurs études ont montré, aprés un antifibrinolytique, un volume de drainage médiastinal moins important mais aucune différence pour le nombre de transfusions ni de concordance avec l'utilisation des PSA. La pertinence de ces études est donc discutable.

Le lecteur doit se poser une deuxième question : l'analyse des données a-t-elle été pertinente ? Nous voulons connaitre la proportion des patients des groupes à l'étude exposés aux PSA; pour ceux qui en ont reçu, la médiane du nombre d'unités administrées et son étendue (il n'est pas approprié d'analyser les résultats avec la moyenne \pm l'écart type, la plupart des produits sanguins hémostatiques étant administrés en quantités prédéterminées, ex. dix unités de plaquettes). Rapporter des résultats de la manière correcte permet de déterminer le risque d'exposition à des PSA et fournit le type d'information que le clinicien peut facilement adapter à la pratique transfusionnelle. ${ }^{3}$ En outre, des statistiques non paramétriques sont, dans la plupart des cas, préférables, étant que donné l'administration des PSA avant et après la chirurgie cardiaque ne suit pas une courbe de distribution symétrique (courbe de Gauss). En effet, la majorité des patients ne reçoivent que peu ou pas de transfusions alors que quelques-uns reçoivent des quantités phénoménales d'hématies et de produits hémostatiques sanguines.

Dans ce numéro du Journal Canadien d'Anesthésie, Dryden et al. décrivent leur expérience avec l'acide tranéxamique administré dans le but de réduire les 
pertes sanguines et le nombre des transfusions de PSA après une chirurgie cardiaque récurrente. En moyenne, la circulation extracorporelle durait près de trois heures et les pertes sanguines étaient abondantes. Dans cette population spécifique, l'acide tranéxamique a effectivement réduit le nombre de transfusions d'hématies, de plasma et de plaquettes. Malheureusement, la présentation des résultats ne permet pas au lecteur de déterminer le pourcentage des patients ayant reçu des PSA, ni le nombre d'unités administrées.

Sachant qu'elle varie amplement d'une institution à une autre, nous devons donc revoir notre stratégie transfusionnelle. ${ }^{4}$ Cette variabilité peut provenir soit parce que les pertes sanguines sont plus importantes dans certains centres, soit parce que les stratégies transfusionnelles sont fautives. Alors que les antifibrinolytiques ne peuvent corriger ces habitudes, on a montré de façons répétées dans plusieurs bonnes études contrôlées qu'ils diminuaient les pertes sanguines et les besoins transfusionnels. ${ }^{5}$ Par contre, quand ce sont les stratégies transfusionnelles qui sont en cause, des mesures correctives ${ }^{6}$ doivent être mises en vigueur avant même de considérer l'emploi des antifibrinolytiques. L'évaluation des pratiques transfusionnelles est une condition sine qua non préalable à l'optimalisation de l'usage des produits sanguins en chirurgie cardiaque.

Finalement, le choix d'un antifibrinolytique spécifique doit être arrêté sur la base de son efficacité, de son influence sur l'incidence des complications majeures et de son coût. Pour sa part, l'efficacité dépendra de la sélection des patients, de la posologie et du moment de son administration. En ce qui concerne le risque d'exposition à des PSA, la plupart des cliniciens s'accordent sur une classification de type «risque faible $v s$ risque élevé», même si cette classification est imparfaite. ${ }^{8}$ L'harmonie est beaucoup plus difficile à réaliser lorsqu'il s'agit de la posologie et de la chronologie de l'administration, essentiellement parce que nous connaissons mal le comportement pharmacocinétique et pharmacodynamique des antifibrinolytiques de synthèse pendant la CEC. Au moment de la mise en marche d'une étude, on a choisi un peu au hasard de les administrer en bolus simples ou répétés de différentes quantités, avec ou sans perfusions subséquentes. La combinaison de ces variables peut susciter un nombre incalculable de protocoles de recherches (sélection des patients, antifibrinolytiques, variabilité posologique, moment de l'administration) et rendre ainsi quasi aléatoire la définition du régime thérapeutique le plus efficace.

La manipulation de l'hémostase comporte certains risques. Pendant quarante-huit heures après la CEC, on constate un état d'hypofibrinolyse caractérisé par des niveaux élevés de PAI-1 (l'inhibiteur de type I de l'activateur du plasminogène). ${ }^{9}$ En favorisant la préservation de clous hémostatiques stables dans les tissus lésés, cet état vraisemblablement bénéfique qu'accentuent les antifibrinolytiques, pourrait théoriquement amplifier le risque de thrombose postopératoire. Paradoxalement, alors que l'aprotinine à haute dose inhibe efficacement la fibrinolyse pendant la $\mathrm{CEC},{ }^{10,11}$ elle n'exagère pas l'interruption tardive du mécanisme de fibrinolyse. ${ }^{9} \mathrm{Au}$ contraire, chez les patients traités à l'aprotinine, la baisse des concentrations de PAI-1 suggère que cet inhibiteur de la protéase pourrait exercer un effet protecteur sur les cellules endothéliales. ${ }^{9}$ On ne connaît pas l'influence des antifibrinolytiques de synthèse sur la fibrinolyse tardive mais l'administration d'acide tranéxamique après la $\mathrm{CEC}$ et la perfusion de protamine a été associée par $\emptyset$ vrum $e t a l^{12}$ à une augmentation inquiétante quoique non significative, de la fréquence de l'infarctus du myocarde postopératoire.

Il est présentement difficile d'évaluer le risque d'accentuation de l'état prothrombotique normal avec les antifibrinolytiques. Cette évaluation s'appuie, après une chirurgie cardiaque, sur l'augmentation, ou le cas échéant, sur l'absence de complications thrombotiques, dont l'infarctus du myocarde, l'occlusion de greffons, les accidents cérébrovasculaires, l'embolie pulmonaire et l'insuffisance rénale. En dépit de la persistance de l'activation de la prothrombine après la $\mathrm{CEC}$, ces accidents sont heureusement peu fréquents. Cependant, faire la preuve qu'il existe des différences dans l'incidence des complications thrombotiques périopératoires requiert malheureusement un nombre considérable de patients. Présentement, ceci n'est possible qu'avec l'aprotinine. Une vaste compilation de données (un total de 2283 cas de chirurgie de revascularisation myocardique) montre que la posologie complète (Hammersmith) est sûre et diminue l'incidence de l'ictus cérébral, mais suggère en même temps que le régime de la demi-dose peut, en réalité, augmenter la mortalité (de $3,8 \%$ à $5,4 \%$ ). ${ }^{14}$ Plus de 3160 patients par groupe auraient été nécessaires pour détecter une augmentation du taux de mortalité de $40 \%$ comme on l'a fait avec le régime de la demi-dose. ${ }^{14}$ Quant aux antifibrinolytiques de synthèse, aucune étude ou compilation d'études publiées jusqu'à maintenant n'a suffisamment de puissance statistique pour exclure l'absence d'influence sur les complications d'origine thrombotique après la chirurgie cardiaque.

L'efficience du traitement est beaucoup plus difficile à définir et à mesurer. Cette question très complexe doit tenir compte non seulement des coûts directs (comme le prix des agents thérapeutiques et des produits sanguins) mais aussi des frais consécutifs à leur utilisation, comme la durée du séjour en salle d'opération, à l'unité des soins intensifs et à l'hôpital ; le coût des révisions chirurgicales et du traitement des complications transfusionnelles et pharmacologiques. Les dépenses occa- 
sionnées par le traitement à l'EACA et à l'AT sont modiques quand on les compare à celles de l'aprotinine. Alors qu'au départ, la modicité des coûts des antifibrinolytique de synthèse constitue un facteur favorable, les conséquences financières des effets secondaires ne doivent pas excéder les économies réalisées. Pour justifier l'utilisation à long terme des antifibrinolytiques, il faut posséder des données suffisantes sur la réduction de la durée des interventions, du temps supplémentaire et du séjour aux soins intensifs ainsi que les preuves des effets avantageux des antifibrinolytiques sur les complications d'origine transfusionnelle (ou autre). Comme nous l'avons mentionné plus haut, l'absence de certaines données nous empêche de prendre une décision éclairée présentement.

Pour revenir à Paracelse, qu'est-ce que ces études nous permettent de conclure ? D'abord, aux doses utilisées présentement, les antifibrinolytique ne sont pas inertes. Deuxièmement, la plupart des études (comme celle de Dryden et al.) tendent à démontrer une activité thérapeutique, dans des cas à haut risque d'hémorragies post-CEC, comme un élément d'une stratégie globale ${ }^{1}$ visant à diminuer l'exposition aux PSA associée à la chirurgie cardiaque. Troisièmement, seules des études réalisées sur une haute échelle permettront d'exclure les effets toxiques des antifibrinolytiques de synthèse. Les risques inhérents aux hémorragies et aux transfusions de PSA doivent être constamment pondérés avec ceux des antifibrinolytiques, spécialement, les accidents thrombotiques. Présentement, l'aprotinine à hautes doses semble la stratégie pharmacologique la moins toxique à notre disposition pour réduire le besoin de PSA après une chirurgie cardiaque sous circulation extracorporelle.

\section{References}

1 Hardy JF, Belisle S, Janvier G, Samama M. Reduction in requirements for allogeneic blood products: nonpharmacologic methods. Ann Thorac Surg 1996; 62: $1935-43$.

2 Janssens $M$, Hartstein $G$, David JL. Reduction in requirements for allogeneic blood products: pharmacologic methods. Ann Thorac Surg 1996; 62: 1944-50.

3 Lemmer JH Jr. Reporting the results of blood conservation studies: the need for uniform and comprehensive methods (Editorial). Ann Thorac Surg 1994; 58 : 1305-6.

4 Goodnough LT, Johnston MFM, Toy PTCY, and the Transfusion Medicine Academic Award Group. The variability of transfusion practice in coronary artery bypass surgery. JAMA 1991; 265: 86-90.

5 Fremes $S E$, Wong BI, Lee E, et al. Metaanalysis of prophylactic drug treatment in the prevention of postoperative bleeding. Ann Thorac Surg 1994; 58: 1580-8.
6 Despotis GJ, Grishaber JE, Goodnough LT. The effect of an intraoperative treatment algorithm on physicians' transfusion practice in cardiac surgery. Transfusion 1994; 34: 290-6.

7 Ferraris VA, Ferraris SP. Limiting excessive postoperative blood transfusion after cardiac procedures. A review. Tex Heart Inst J 1995; 22: 216-30.

8 Belisle $S$, Hardy JF. Hemorrhage and the use of blood products after adult cardiac operations: myths and realities. Ann Thorac Surg 1996; 62: 1908-17.

$9 \mathrm{Lu} \mathrm{H}, \mathrm{Du}$ Buit C, Soria J, et al. Postoperative hemostasis and fibrinolysis in patients undergoing cardiopulmonary bypass with or without aprotinin therapy. Thromb Haemost 1994; 72: 438-43.

10 Marx G, Pokar H, Reuter $H$, Doering V, Tilsner V. The effects of aprotinin on hemostatic function during cardiac surgery. J Cardiothorac Vasc Anesth 1991; 5: 467-74.

11 Blaubut B, Gross C, Necek S, Doran JE, Späth $P$, Lundsgaard-Hansen $P$. Effects of high-dose aprotinin on blood loss, platelet function, fibrinolysis, complement, and renal function after cardiopulmonary bypass. $\mathrm{J}$ Thorac Cardiovasc Surg 1991; 101: 958-67.

12 Øorum E, Am Holen E, Abdelnoor $M$, Øystese $R$, Ringdal $M L$. Tranexamic acid (Cyklokapron) is not necessary to reduce blood loss after coronary artery bypass operations. J Thorac Cardiovasc Surg 1993; 105: 78-83.

13 Slaughter TF, LeBleu TH, Douglas JM Jr, Leslie JB, Parker JK, Greenberg CS. Characterization of prothrombin activation during cardiac surgery by hemostatic molecular markers. Anesthesiology 1994; 80 : 520-6.

14 Smith PK, Mublbaier LH. Aprotinin: safe and effective only with the full-dose regimen (Editorial). Ann Thorac Surg 1996; 62: 1575-7. 